\title{
Equivalence of topical clobetasone and dexamethasone in experimental corneal allograft rejection
}

\author{
KIRK R. Wilhelmus, PAUl A. HUNTER, and NOEL S. C. RiCE \\ From the Pocklington Eye Transplantation Research Unit, Institute of Ophthalmology, London
}

SUMMARY We produced experimental immune reactions by exchanging peripheral corneal transplants between rabbits. Clobetasone butyrate $0.1 \%$ and dexamethasone phosphate $0.1 \%$ eye drops were equally effective in delaying corneal allograft rejection.

Allograft rejection is a leading cause of corneal transplant failure. Studies in rabbits have demonstrated the use of corticosteroids in the prevention of these immune responses. ${ }^{12}$ Because a new steroid clobetasone butyrate may have effective antiinflammatory and immunosuppressive properties ${ }^{3}$ we have compared it with dexamethasone in an experimental model of penetrating keratoplasty.

\section{Material and methods}

We performed penetrating keratoplasties on pairs of outbred Dutch pigmented and New Zealand white rabbits weighing $1-2.5 \mathrm{~kg}$. Anaesthesia was induced with intravenous pentobarbitone sodium. Each animal then received amethocaine $1 \%$, cyclopentolate $\mathrm{HC} 11 \%$, and phenylephrine $\mathrm{HC} 110 \%$ topically and 2500 units $/ \mathrm{kg}$ heparin intravenously. Under operating microscopes peripheral $6 \mathrm{~mm}$ corneal buttons were exchanged between pigmented and albino rabbits; the grafts were positioned eccentrically 1-2 mm from the corneoscleral limbus and secured with a continuous $10-0$ monofilament nylon suture. Each animal received atropine $1 \%$ and chloramphenicol $0.5 \%$ eyedrops postoperatively.

Twenty animals were randomly assigned to one of 2 treatment groups. Group 1 received dexamethasone sodium phosphate $0 \cdot 1 \%$ solution; group 2 received clobetasone butyrate $0 \cdot 1 \%$ suspension (Eumovate). Treatment was begun at the conclusion of surgery and continued 4 times daily for 14 days. Sutures were then removed, and treatment was given twice daily for a

Correspondence to Mr N. S. C. Rice, Professorial Unit, Moorfields Eye Hospital, City Road, London EC1. subsequent 14 days and then discontinued. Twelve additional animals received only topical atropine and chloramphenicol drops postoperatively as a control group. Only animals in which a successful corneal graft cleared within 2 days were included in the study.

We performed biomicroscopic slit-lamp examinations of each animal at alternate-day intervals. The onset of the allograft reaction was judged to occur when one or more of the following were first noted at the peripheral edge of the graft: an epithelial rejection line (Fig. 1), a whitish band of stromal infiltrate, or a line of keratic precipitates on the endothelium with adjacent stromal oedema. ${ }^{4}$

We compared the treatments by constructing actuarial survival curves to the onset of rejection and used Student's $t$ test and the logrank test to assess statistical differences.

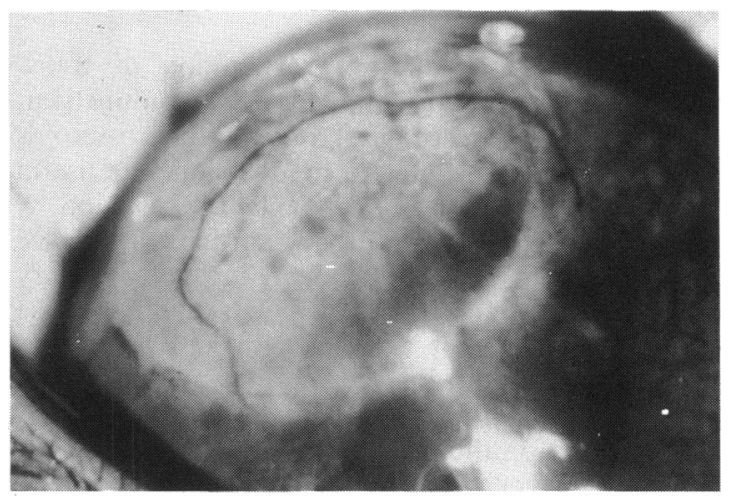

Fig. 1 Epithelial rejection line stained with methylene blue in a peripheral corneal transplant. 
Fig. 2 Survival tables for allograft rejection-free duration of peripheral corneal grafts. Difference between control group and 2 treatment groups is significant $(p<0.05)$; no statistical difference is present between topical steroid groups. Arrow, average day of initial graft vascularisation; shaded area, treatment period.

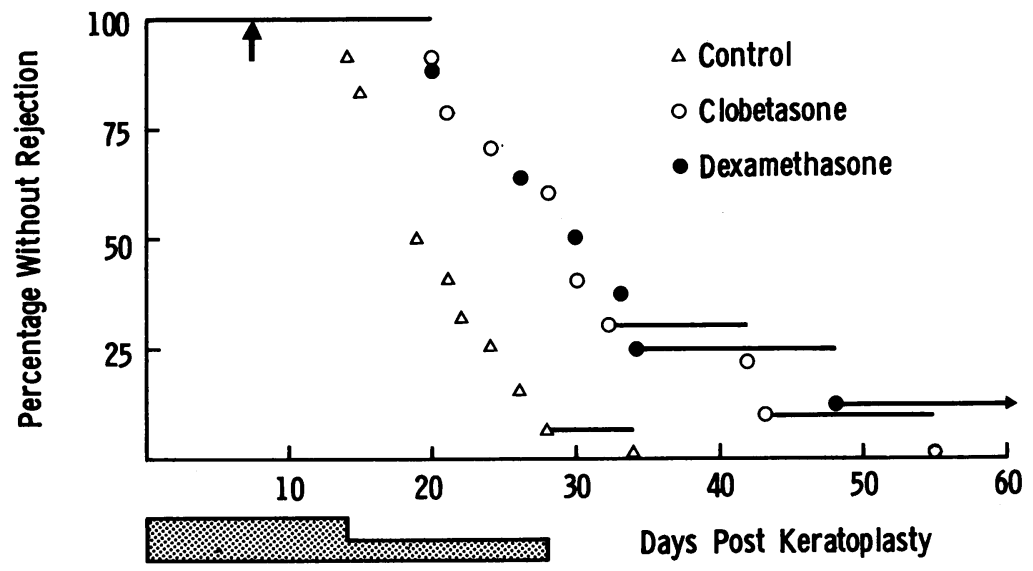

fore compared it with dexamethasone in rabbits undergoing corneal transplantation.

Because central penetrating corneal allografts in avascular rabbit corneas are not consistently rejected, the immune reaction produced by an eccentrically placed graft $^{4}$ provides a more reliable model for assessing topical immunosuppressive drugs. ${ }^{7}$ Vascularisation of the graft occurs at an average of $7 \cdot 5$ days after transplantation and rejection 1 to 3 weeks later. Topical steroids were shown to delay the onset of rejection; clobetasone and dexamethasone were -equally effective at the dosage regimen used. The occurrence of an immune reaction after dosage reduction from 4 to 2 times per day suggests that twice-daily use is insufficient to prevent rejection in this experimental model. Prolonged and more frequent applications are necessary to determine if complete inhibition of graft rejection can be achieved.

As a result of improvements in surgical technique and preservation of donor material graft rejection remains the leading cause of late failure of corneal transplants. Extended steroid use may be required to control an immune reaction despite the risk of deleterious side effects. Clobetasone butyrate may offer a useful alternative to dexamethasone following corneal transplantation in patients genetically predisposed to steroid-induced glaucoma.

This study was supported by a grant from the Board of Governors, Moorfields Eye Hospital, and by a fellowship from the Friends of Moorfields Eye Hospital (Dr Wilhelmus).

Dr W. Ng provided technical assistance. occur, steroids with limited intraocular penetration such as fluorometholone have been evaluated in the prevention of immune graft rejection. ${ }^{2}$ Clobetasone butyrate is a recently developed steroid which is clinically effective in decreasing ocular inflammation with minimal effect on intraocular pressure. ${ }^{36}$ We there- 
2 Fleming JC. Reid FR. Wood TO. Prevention of immune graft rejection after corneal transplantation. Am J Ophthalmol 1979;88: 97-101.

3 Ramsell TG. Bartholomew RS. Walker SR. Clinical evaluation of clobetasone butyrate: a comparative study of its effects in postoperative inflammation and on intraocular pressure. $\mathrm{Br} J$ Ophthalmol 1980; 64: 43-5.

4 Silverstein AM, Khodadoust AA. Transplantation immunobiology of the cornea. In: Corneal Graft Failure. Ciba Foundation Symposium 15. Amsterdam: Associated Scientific Publishers. 1973: 105-25.
5 Jones BR. Present knowledge and problems of corneal graft failure. In: Corneal Graft Failure. Amsterdam: Associated Scientific Publishers, 1973: 349-54.

6 Buckley CA. Brown AJ. Leonard MC. The ocular penetration and clinical use of clobetasone butyrate. In: Trevor-Roper P, ed. The Cornea in Health and Disease. VIth Congress of the European Society of Ophthalmology. London: Academic Press, 1981: 349-56.

7. Hunter PA. Wilhelmus KR. Rice NSC. Jones BR. Cyclosporin A applied topically to the recipient eye inhibits corneal graft rejection. Clin Exp Immunol in press. 